\title{
ON SPECTRAL PROPERTIES OF PERTURBED OPERATORS
}

\author{
M. THAMBAN NAIR
}

(Communicated by Palle E. T. Jorgensen)

\begin{abstract}
Farid (1991) has given an estimate for the norm of a perturbation $V$ required to obtain an eigenvector for the perturbed operator $T+V$ within a given ball centered at a given eigenvector of the unperturbed (closed linear) operator $T$. A similar result is derived from a more general result of the author (1989) which also guarantees that the corresponding eigenvalue is simple and also that the eigenpair is the limit of a sequence obtained in an iterative manner.
\end{abstract}

\section{INTRODUCTION}

In a recent paper [3] Farid has considered a method based on contraction mapping theorem instead of the fixed-point theorem approach of Rosenbloom [7] to address the following problem in perturbation theory:

If $\left(\lambda_{0}, \phi_{0}\right)$ is an eigenpair of a densely defined closed linear operator in a Banach space $\mathbf{X}$, and $r$ and $\rho$ are given positive reals, then obtain an estimate for the radius of the disc $\{V \in B L(X):\|V\| \leq \delta\}$ such that the perturbed operator $T+V$ has an eigenpair $(\lambda, \phi)$ with

$$
\left\|\phi-\phi_{0}\right\| \leq r \text { and }\left|\lambda-\lambda_{0}\right| \leq \rho
$$

for every $V$ in $\{V \in B L(X):\|V\| \leq \delta\}$, where $B L(X)$ denotes the space of all bounded linear operators on $X$.

In this note a result similar to that of Farid [3] is derived from a more general result in Nair [5]. While the results of Farid [3] and Rosenbloom [7] are essentially existential results, ours is an iterative procedure where sequences $\left(\lambda_{k}\right)$ and $\left(\phi_{k}\right)$ are obtained in an iterative manner with the property that $\lambda_{k} \rightarrow \lambda$ and $\phi_{k} \rightarrow \phi$ as $k \rightarrow \infty$. Moreover, the eigenvalue $\lambda$ is shown to be a simple eigenvalue of $T+V$, and a disc centered at $\lambda_{0}$ is obtained where $\lambda$ is the only spectral value of $T+V$ lies. The uniqueness of the pair $(\lambda, \phi)$ established by Farid [3] is a consequence of the simplicity of $\lambda$.

\section{THE MAIN RESULT}

Let $T$ be a closed linear operator in a Banach space $X$ with a dense domain $D$. Let $\lambda_{0}$ be an eigenvalue of $T$ with a corresponding eigenvector $\phi_{0}$ with

Received by the editors August 16, 1993 and, in revised form, October 15, 1993.

1991 Mathematics Subject Classification. Primary 47A55, 47A10. 
$\left\|\phi_{0}\right\|=1$. The basic assumption in Farid [3] is the following:

(i) $\lambda_{0}^{*}$, the complex conjugate of $\lambda_{0}$, is an eigenvalue of the adjoint operator $T^{*}$, and $\phi_{0}^{*} \in X^{*}$ is a corresponding eigenvector such that $\left\langle\phi_{0}, \phi_{0}^{*}\right\rangle=1$.

(ii) $\lambda_{0}$ does not belong to the spectrum of the operator $\tilde{T}:=\left.T\right|_{Y}$, where $Y=\left\{x \in D:\left\langle x, \phi_{0}^{*}\right\rangle=0\right\}$.

Here and in what follows $X^{*}$ denotes the adjoint space of $X$, that is, the space of all conjugate linear functionals on $X$, and $\langle x, f\rangle$ denotes the complex conjugate of $f(x)$ for $x \in X$ and $x^{*} \in X^{*}$. The adjoint operator $T^{*}$ is defined by $\left\langle x, T^{*} f\right\rangle=\langle T x, f\rangle$ for all $x \in D$ and $f \in D\left(T^{*}\right):=\left\{f \in X^{*}\right.$ : there exists $g \in X^{*}$ with $\langle T x, f\rangle=\langle x, g\rangle$ for all $\left.x \in D\right\}$. First we observe that assumption (i) implies the subspaces

$$
X_{1}:=\left\{x \in X:\left\langle x, \phi_{0}^{*}\right\rangle \phi_{0}=x\right\}
$$

and

$$
X_{2}:=\left\{x \in X:\left\langle x, \phi_{0}^{*}\right\rangle=0\right\}
$$

are invariant under $T$, i.e., $T x \in X_{i} \cap D$ for every $x \in X_{i} \cap D, i=1,2$, with

$$
X=X_{1} \oplus X_{2},
$$

and assumption (ii) implies, as a consequence of Theorem 4.2 in Nair [5], that $\lambda$ is in fact a simple eigenvalue of $T$. Also, we note that the operator $P_{0}: X \rightarrow X$ defined by

$$
P_{0} x=\left\langle x, \phi_{0}^{*}\right\rangle \phi_{0}, \quad x \in X,
$$

is the projection operator onto $X_{1}$ along $X_{2}$, and $\left\|P_{0}\right\|=\left\|\phi_{0}^{*}\right\|$. Let

$$
S_{0}:=\left(\tilde{T}-\lambda_{0}\right)^{-1}: X_{2} \rightarrow X_{2} \text {. }
$$

With the above notation the main result of Farid [3] is the following.

Theorem (Farid [3, Theorem 2.1]). For every real number $r$ satisfying

$$
0<r<\left(\frac{\left\|S_{0}\left(I-P_{0}\right)\right\|}{\left\|P_{0}\right\|\left\|S_{0}\right\|}\right)^{1 / 2}
$$

and every bounded linear operator $V$ on $X$ satisfying

$$
\|V\| \leq \delta(r):=r /\left(\left\|P_{0}\right\|\left\|S_{0}\right\| r^{2}+\left(\left\|P_{0}\right\|\left\|S_{0}\right\|+\left\|S_{0}\left(I-P_{0}\right)\right\|\right) r+\left\|S_{0}\left(I-P_{0}\right)\right\|\right),
$$

the operator $T+V$ has a unique eigenpair $(\lambda, \phi)$ such that

$$
\left\langle\phi, \phi_{0}^{*}\right\rangle=1, \quad\left\|\phi-\phi_{0}\right\| \leq r,
$$

and

$$
\left|\lambda-\lambda_{0}\right| \leq\|V\|\left(1+\left\|\phi-\phi_{0}\right\|\right)\left\|P_{0}\right\| .
$$

The main result of this note is the following.

Theorem * . For every real number $r>0$ and for every bounded linear operator $V$ on $X$ satisfying

$$
\beta_{V}:=\max \left\{\left\|P_{0} V\right\|,\left\|\left(I-P_{0}\right) V\right\|\right\} \leq \frac{r}{\left\|S_{0}\right\|(1+r)^{2}},
$$

the operator $T+V$ has a simple eigenvalue $\lambda$ and a corresponding (unique) 
eigenvector $\phi$ such that

$$
\begin{gathered}
\left\langle\phi, \phi_{0}^{*}\right\rangle=1, \quad\left\|\phi-\phi_{0}\right\| \leq r, \\
\left|\lambda-\lambda_{0}\right| \leq\|V\|\left(\left\|\phi-\phi_{0}\right\|+1\right)\left\|P_{0}\right\|,
\end{gathered}
$$

and $\lambda$ is the only spectral value of $T+V$ lying in the disc

$$
\Delta_{0}:=\left\{z:\left|z-q_{0}\right|<\frac{d_{0}}{2}(1+\sqrt{1-4 \mu})\right\}
$$

where

$$
d_{0}=\frac{\left(1-2 \beta_{V}\left\|S_{0}\right\|\right)}{\left\|S_{0}\right\|}, \quad q_{0}=\lambda_{0}+\left\langle V \phi_{0}, \phi_{0}^{*}\right\rangle, \quad \mu=\left(\frac{r}{1+r^{2}}\right)^{2} .
$$

Moreover,

$$
\lambda=\lim _{k \rightarrow \infty} \lambda_{k}, \quad \phi=\lim _{k \rightarrow \infty} \phi_{k},
$$

where $\lambda_{k}$ and $\phi_{k}$ are defined iteratively as

$$
\begin{aligned}
\left(\tilde{T}+\tilde{V}-\lambda_{0}-\left\langle V \phi_{0}, \phi_{0}^{*}\right\rangle\right) \psi_{1} & =-\left(I-P_{0}\right) V \phi_{0}, \\
\phi_{1} & =\phi_{0}+\psi_{1}, \\
\lambda_{1} & =\lambda_{0}+\left\langle V \psi_{1}, \phi_{0}^{*}\right\rangle
\end{aligned}
$$

and, for $k=1,2, \ldots$,

$$
\begin{aligned}
\left(\widetilde{T}+\widetilde{V}-\lambda_{0}-\left\langle V \phi_{0}, \phi_{0}^{*}\right\rangle\right) x_{k} & =\left\langle V \psi_{k}, \phi_{0}^{*}\right\rangle \psi_{k}, \\
\psi_{k+1} & =\psi_{1}+x_{k}, \\
\phi_{k+1} & =\phi_{0}+\psi_{k+1}, \\
\lambda_{k+1} & =\lambda_{0}+\left\langle V \psi_{k+1}, \phi_{0}^{*}\right\rangle .
\end{aligned}
$$

Here $\widetilde{T}=\left.T\right|_{Y}$ and $\tilde{V}=\left(I-P_{0}\right) V_{\left(I-P_{0}\right) X}$.

Remark. We note that

$$
\beta_{V}:=\max \left\{\left\|P_{0} V\right\|,\left\|\left(I-P_{0}\right) V\right\|\right\} \leq c_{0}\|V\|,
$$

where $c_{0}=\max \left\{\left\|P_{0}\right\|,\left\|I-P_{0}\right\|\right\}$. Therefore, a sufficient condition for Theorem ${ }^{*}$ to hold is

$$
\|V\| \leq \omega(r):=\frac{r}{c_{0}\left\|S_{0}\right\|(1+r)^{2}} .
$$

Also,

$$
\left\|P_{0}\right\|\left\|S_{0}\right\| r^{2}+\left(\left\|P_{0}\right\|\left\|S_{0}\right\|+\left\|S_{0}\left(I-P_{0} \|\right) r+\right\| S_{0}\left(I-P_{0}\right)\left\|\leq c_{0}\right\| S_{0} \|(1+r)^{2},\right.
$$

so that in general,

$$
\omega(r) \leq \delta(r),
$$

and thereby the assumption ' $\|V\| \leq \delta(r)$ ' of Farid [3] is weaker than ' $\|V\| \leq$ $\omega(r)$ '. However, if $\left\|P_{0}\right\|=1=\left\|I-P_{0}\right\|$, then

$$
\beta_{V} \leq\|V\|, \quad \omega(r)=\delta(r)=\frac{r}{\left\|S_{0}\right\|(1+r)^{2}},
$$

so in this case the condition in Theorem ${ }^{*}$ is weaker than that of Farid [3], and therefore Theorem ${ }^{*}$ improves the result of Rosenbloom [7] also. Examples with $\beta_{V}<\|V\|$ can be easily constructed. It is to be noted that if $X$ is a 
Hilbert space and $T$ is a normal operator on $X$, then we have $\phi_{0}^{*}=\phi_{0}$, so that the projection $P_{0}$ is orthogonal and therefore $\left\|P_{0}\right\|=1=\left\|I-P_{0}\right\|$.

We recall the following from [5] or [4]. If $X=Y_{1} \oplus Y_{2}$ is a decomposition of $X$ into closed subspaces $Y_{1}$ and $Y_{2}, B$ is a bounded linear operator on $Y_{1}$, and $C$ is a closed linear operator in $Y_{2}$ with domain $D_{C}$, then the operator $F: B L\left(Y_{1}, Y_{2} \cap D_{C}\right) \rightarrow B L\left(Y_{1}, Y_{2}\right)$ defined by

$$
F(K)=C K-K B, \quad K \in B L\left(Y_{1}, Y_{2} \cap D_{C}\right)
$$

has a bounded inverse on $B L\left(Y_{1}, Y_{2}\right)$ if and only if $\sigma(B) \cap \sigma(C)=\varnothing$. The separation between $B$ and $C$ is defined by

$$
\operatorname{sep}(B, C):= \begin{cases}1 /\left\|F^{-1}\right\| & \text { if } F \text { has bounded inverse } \\ 0 & \text { otherwise. }\end{cases}
$$

If $E_{1} \in B L\left(Y_{1}\right)$ and $E_{2} \in B L\left(Y_{2}\right)$, then

$$
\operatorname{sep}\left(B+E_{1}, C+E_{2}\right) \geq \operatorname{sep}(B, C)-\left(\left\|E_{1}\right\|+\left\|E_{2}\right\|\right) \text {. }
$$

Proof (Theorem ${ }^{*}$ ). Let $\left(T_{i j}\right),\left(V_{i j}\right)$, and $\left(A_{i j}\right), i, j=1,2$, be the $2 \times 2$ matrix representations of $T, V$, and $A=T+V$ respectively with respect to the decomposition $X=X_{1} \oplus X_{2}$ (cf. [8, p. 286]). Then it is seen that

$$
\left\|V_{i j}\right\| \leq\left\|P_{i} V\right\| \leq \beta_{V}:=\max \left\{\left\|P_{0} V\right\|,\left\|\left(I-P_{0}\right) V\right\|\right\}, \quad i, j=1,2,
$$

with $P_{1}=P_{0}$ and $P_{2}=I-P_{0}$. Therefore, we have

$$
\operatorname{sep}\left(A_{11}, A_{22}\right) \geq \operatorname{sep}\left(T_{11}, T_{22}\right)-\left(\left\|V_{11}\right\|+\left\|V_{22}\right\|\right) \geq \frac{\left(1-2 \beta_{V}\left\|S_{0}\right\|\right)}{\left\|S_{0}\right\|} .
$$

Now the condition $\beta_{V} \leq r /(1+r)^{2}\left\|S_{0}\right\|$ implies that $2 \beta_{V}\left\|S_{0}\right\| \leq 2 r /(1+r)^{2} \leq \frac{1}{2}$, so that $\operatorname{sep}\left(A_{11}, A_{22}\right)>0$ and consequently the assumption $\sigma\left(A_{11}\right) \cap \sigma\left(A_{22}\right)=$ $\varnothing$ in Nair [5] is satisfied. Now the quantity $\varepsilon$ in [5] is seen to satisfy

$$
\begin{aligned}
\varepsilon & :=\frac{\left\|F^{-1}\left(A_{12}\right)\right\|\left\|A_{12}\right\|}{\operatorname{sep}\left(A_{11}, A_{22}\right)} \leq \frac{\left\|A_{12}\right\| A_{21} \|}{\operatorname{sep}\left(A_{11}, A_{22}\right)^{2}} \\
& \leq\left(\frac{\beta_{V}\left\|S_{0}\right\|}{1-2 \beta_{V}\left\|S_{0}\right\|}\right)^{2} \leq\left(\frac{r}{1+r^{2}}\right)^{2} \leq \frac{1}{4}
\end{aligned}
$$

Writing $\mu=\left(r /\left(1+r^{2}\right)\right)^{2}$ and $g(\mu)=(1-\sqrt{1-4 \mu}) / 2 \mu$, it follows from ([5, Theorem 4.3 and relation (4.4)]) that $A:=T+V$ has a simple eigenvalue $\lambda$ and a corresponding eigenvector $\phi$ such that

$$
\begin{aligned}
\left\langle\phi, \phi_{0}^{*}\right\rangle & =1 \\
\left\|\phi-\phi_{0}\right\| & \leq \alpha g(\mu), \\
\left|\lambda-\lambda_{0}\right| & \leq \frac{\delta_{0}}{2}(1-\sqrt{1-4 \mu})
\end{aligned}
$$

and $\lambda$ is the only spectral value of $A$ lying in the disc

$$
\left\{z: z-\lambda_{0} \mid<\frac{\delta_{0}}{2}(1+\sqrt{1-4 \mu})\right\} \supseteq \Delta_{0} \text {. }
$$

Here

$$
\begin{gathered}
\delta_{0}:=\operatorname{sep}\left(A_{11}, A_{22}\right) \geq \frac{\left(1-2 \beta_{V}\left\|S_{0}\right\|\right)}{\left\|S_{0}\right\|}=d_{0}, \\
\alpha \leq \frac{\left\|\left(I-P_{0}\right) V\right\|}{\operatorname{sep}\left(A_{11}, A_{22}\right)} \leq \frac{\beta_{V}\left\|S_{0}\right\|}{1-2 \beta_{V}\left\|S_{0}\right\|} \leq \frac{r}{1+r^{2}}=\sqrt{\mu},
\end{gathered}
$$


and $g(t), 0<t \leq \frac{1}{4}$, satisfies

$$
\begin{gathered}
1 \leq g(t) \leq 2, \\
g\left(t_{1}\right) \leq g\left(t_{2}\right) \text { for } t_{1} \leq t_{2}, \\
\lim _{t \rightarrow 0} g(t)=1, \text { and } \lim _{t \rightarrow 1 / 4} g(t)=2 .
\end{gathered}
$$

It is easily seen that

$$
\alpha g(\mu) \leq \sqrt{\mu} g(\mu) \leq r,
$$

so that $\left\|\phi-\phi_{0}\right\| \leq r$. Since $\left\langle\phi, \phi_{0}^{*}\right\rangle=1$ and $T^{*} \phi_{0}^{*}=\lambda_{0}^{*} \phi_{0}^{*}$, we have

$$
\lambda=\lambda_{0}+\left\langle V\left(\phi-\phi_{0}\right), \phi_{0}^{*}\right\rangle+\left\langle V \phi_{0}, \phi_{0}^{*}\right\rangle \text {. }
$$

Therefore,

$$
\left|\lambda-\lambda_{0}\right| \leq \beta_{V}\left(\left\|\phi-\phi_{0}\right\|+1\right)\left\|P_{0}\right\| \text {. }
$$

If $\tilde{\phi}$ is another eigenvector of $T+V$ corresponding to the simple eigenvalue $\lambda$ such that $\left\langle\tilde{\phi}, \phi_{0}^{*}\right\rangle=1$, then $\tilde{\phi}=c \phi$ for some constant $c \neq 0$, and therefore $1=\left\langle\tilde{\phi}, \phi_{0}^{*}\right\rangle=c\left\langle\phi, \phi_{0}^{*}\right\rangle=c$. Thus $\tilde{\phi}=\phi$, proving the uniqueness of $\phi$.

Lastly, the iterative procedure to obtain $\left(\lambda_{k}\right)$ and $\left(\phi_{k}\right)$, and their convergence to $\lambda$ and $\phi$ respectively, are the consequences of [5, relations (3.5), (3.6)] and [5, Theorem 4.3], respectively.

Remark. We note that the generalized Rayleigh quotient $q=\left\langle(T+V) \phi_{0}, \phi_{0}^{*}\right\rangle$ of $T+V$ at $\left(\phi_{0}, \phi_{0}^{*}\right)$ satisfies

$$
|\lambda-q| \leq \beta_{V}\left\|\phi-\phi_{0}\right\| \text {. }
$$

A similar reformulation of the results in Nair [5, 6] and Stewart [9] involving spectral sets and spectral subspaces will show their applicability to more general situations of diagonally dominant infinite matrices than the ones described in [1-3].

\section{ACKNOWLEDGMENT}

This work was done during the author's visit to the Centre for Mathematics and Its Applications, The Australian National University, Canberra, during June-December 1993. The support received and the useful discussions he had with Dr. R. S. Anderssen are gratefully acknowledged.

\section{REFERENCES}

1. F. O. Farid and P. Lancaster, Spectral properties of diagonally dominant infinite matrices, Part I, Proc. Roy. Soc. Edinburgh Sect. A 111 (1989), 301-314.

2. F. O. Farid, Spectral propereties of diagonally dominant infinite matrices, Part II, Linear Algebra Appl. 143 (1991), 7-17.

3. _ Spectral properties of perturbed linear opeerators and their application to infinite matrices, Proc. Amer. Math. Soc. 112 (1991), 1013-1022.

4. M. T. Nair, Approximation and localization of eigenelements, Ph.D. Thesis, I. I. T. Bombay, 1984.

5. __ Approximation of spectral sets and spectral subspaces in Banach spaces, J. Indian Math. Soc. (N.S.) 54 (1989), 187-200.

6. __ On iterative refinements for spectral sets and spectral subspaces, Numer. Funct. Anal. Optim. 10 (1989), 1019-1037. 
7. P. Rosenbloom, Perturbation of linear operators in Banach spaces, Arch. Math. 6 (1955), 89-101.

8. A. E. Taylor and D. C. Lay, Introduction to functional analysis, Wiley, New York, 1980.

9. G. W. Stewart, Error bounds for approximate invariant subspaces of closedlinear operators, SIAM J. Numer. Anal. 8 (1971), 796-808.

Department of Mathematics, GoA University, GoA - 403 203, India 